\title{
Neutron capture on the s-process branch point nucleus ${ }^{63} \mathrm{Ni}$
}

\section{Weigand ${ }^{* a}$, T. A. Bredeweg ${ }^{c}$, A. Couture ${ }^{c}$, M. Jandel ${ }^{c}$, F. Käppeler ${ }^{b}$, C. Lederer ${ }^{a}$, G. Korschinek ${ }^{d}$, M. Krticka ${ }^{e}$, J. M. O'Donnell ${ }^{c}$, R. Reifarth ${ }^{a}$, J. Ullmann ${ }^{c}$, A. Wallner ${ }^{f}$,} E-mail: m.weigandegsi.de

${ }^{a}$ Goethe University, Frankfurt, Germany

${ }^{b}$ FZK, Karlsruhe, Germany

${ }^{c}$ LANL, Los Alamos, USA

${ }^{d}$ TUM, Garching, Germany

${ }^{e}$ Charles University, Czech Republic

${ }^{f}$ ANU, Australia

\begin{abstract}
The cosmic abundance distribution of elements and isotopes is related to the reaction rates of the different synthesis processes. It is important to know those rates under the different conditions found in the universe to explain the origin of this abundance pattern. Most of the elements heavier than iron have been and still are synthesized in neutron-induced reactions in stars of different stages. Nearly all of the observed abundances are either formed by the s- or the r-process in almost equal shares. Of special interest are branching point nuclei like ${ }^{63} \mathrm{Ni}$, where the s-process path can take different ways. The neutron capture cross (CS) section of ${ }^{63} \mathrm{Ni}$ affects the abundances of other nuclei in this region. In order to determine the energy dependent neutron capture cross section in the astrophysical energy region $\left(\mathrm{kT}_{\text {astro }} \approx 1 \ldots 100 \mathrm{keV}\right)$, an experiment at the Los Alamos National Laboratory has been performed using the calorimetric $4 \pi \mathrm{BaF}_{2}$ array DANCE. A preliminary result of the ${ }^{63} \mathrm{Ni}$ experiment is presented. The (n, $\gamma$ ) CS of ${ }^{63} \mathrm{Ni}$ has been determined relative to the known ${ }^{62} \mathrm{Ni} \mathrm{CS}$, which was also measured at Los Alamos [1]. Various possible ${ }^{63} \mathrm{Ni}$ resonances have been identified via the Q-value. The contribution of other isotopes in the sample could be identified.
\end{abstract}

XII International Symposium on Nuclei in the Cosmos

August 5-12, 2012

Cairns, Australia

${ }^{*}$ Speaker. 


\section{Introduction}

One of the important questions in nuclear astrophysics is how the observed abundances of nuclei came to be. For this reason, it is important to know the rates of all relevant reactions under the different conditions found in the universe. Most of the elements heavier than iron have been and still are synthesized in neutron-induced reactions in stars of different stages. Nearly all of the observed abundances are either formed by the s- or the r-process in almost equal shares.

The precise s-process path depends on parameters like temperature, neutron density, cross sections (CS) and half-lifes in case of unstable isotopes. Some of those unstable isotopes on the s-process path play a special role. They act as branching points, creating different possible ways for the nucleosynthesis, neutron capture and $\beta$-decay compete. This branching affects the isotopic abundances of the heavier elements in the weak s-process component [3] [4]. Therefore it is important to know the capture CS for these isotopes. ${ }^{63} \mathrm{Ni}$ with $\mathrm{t}_{1 / 2} \approx 100 \mathrm{yrs}$ is one of these branching points and there is no experimentel CS data so far. Figure 1 shows the current situation. Network calculations show a sensitivity on the ${ }^{63} \mathrm{Ni}$ CS for some nuclei in the vicinity of Nickel. A deviation from the theoretical predictions can affect the abundances of ${ }^{64} \mathrm{Ni}$, the s-only nucleus ${ }^{64} \mathrm{Zn}$, and the isotopic ratio of ${ }^{63} \mathrm{Cu}$ and ${ }^{65} \mathrm{Cu}$.

In order to determine the energy dependent neutron capture cross section in the astrophysical energy region $\left(\mathrm{kT}_{\text {astro }} \approx 1 \ldots 100 \mathrm{keV}\right.$ ), an experiment at the Los Alamos National Laboratory has been performed using the calorimetric $4 \pi \mathrm{BaF}_{2}$ array DANCE [5]. A preliminary result of the ${ }^{63} \mathrm{Ni}$ experiment is presented. The $(\mathrm{n}, \gamma) \mathrm{CS}$ of ${ }^{63} \mathrm{Ni}$ has been determined relative to the known ${ }^{62} \mathrm{Ni} \mathrm{CS}$, which was also measured at Los Alamos [1]. Various possible ${ }^{63} \mathrm{Ni}$ resonances have been identified via the Q-value. The contribution of other isotopes in the sample could be identified.

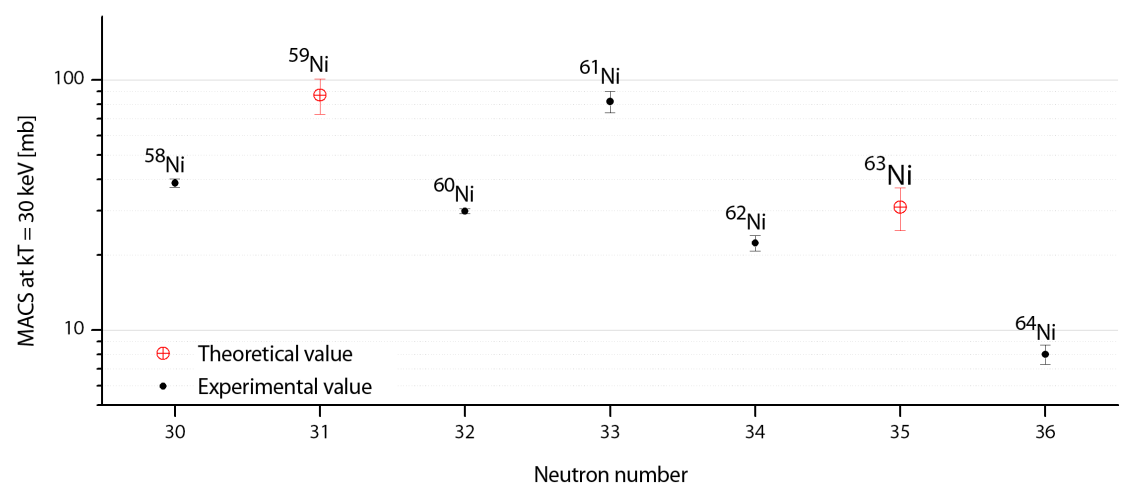

Figure 1: Experimental (black) and theoretical (red) MACS (30 keV) for the Nickel isotopes from the KADoNiS database. [2] 

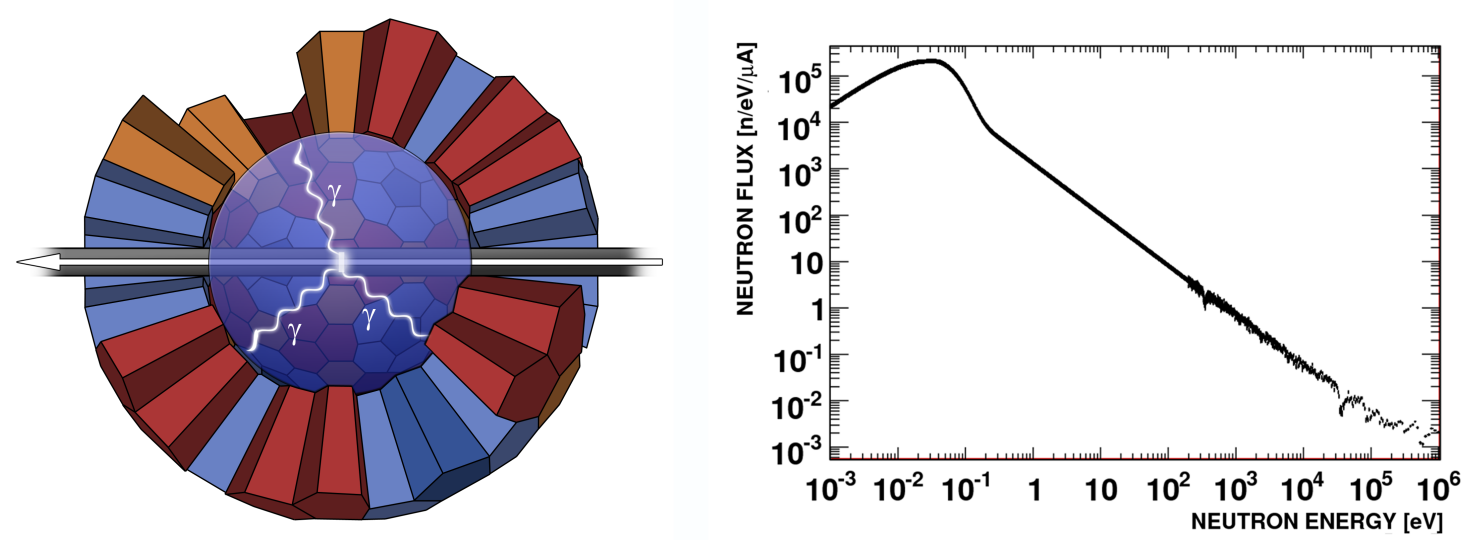

Figure 2: Left: Schematic drawing of the $4 \pi \mathrm{BaF}_{2}$-detector geometry. The neutrons come from the right side; the sample position is in the center. The semi-transparent sphere depicts the $\mathrm{LiH}_{6}$ neutron absorber. Right: The neutron flux energy spectrum at the DANCE experimental site.

\section{The DANCE array}

At the Los Alamos Neutron Science CEnter (LANSCE) prompt $\gamma$-rays from ${ }^{63} \mathrm{Ni}(\mathrm{n}, \gamma)$ capture events have been observed with the Detector for Advanced Neutron Capture Experiments (DANCE). DANCE is a $4 \pi \mathrm{BaF}_{2}$-detector, which consists of 160 spherically arranged $\mathrm{BaF}_{2}-$ crystals (Figure 2). The inner radius of the sphere is $34 \mathrm{~cm}$. The space between the beam pipe and the crystals is filled with a $\mathrm{LiH}_{6}$ spherical shell, in order to reduce background from scattered neutrons. This is very important, since capture CS are usually smaller than for scattering. DANCE is a high efficiency detector for $\gamma$-cascades able to run in a calorimetric mode. The data aquisition is running with an readout rate of $500 \mathrm{MHz}$. The system delivers several information:

- Total $\gamma$ energy radiated after capture $\left(E_{t o t}\right)$.

- Angular distribution.

- Multiplicity $(M)$.

- Neutron energy via time-of-flight (TOF) methode; the flight path length is $20 \mathrm{~m}$.

\section{The sample}

The sample, a metallic foil of $10.5 \mathrm{~cm}$ in diameter, was produced more than 20 years ago by neutron irradiation of an enriched ${ }^{62} \mathrm{Ni}$ sample. The irradiation resulted in about $11 \%$ of ${ }^{63} \mathrm{Ni}$. The total mass of the sample is $347 \mathrm{mg}$. Since much time passed, one could expect a considerable amount of the decay product ${ }^{63} \mathrm{Cu}$. Follow-up investigations at PSI in Switzerland showed that the sample contained about $5.94 \mathrm{mg}$ of ${ }^{63} \mathrm{Cu}$. Additionally ${ }^{59} \mathrm{Ni}$ was produced during the irradiation as well. 


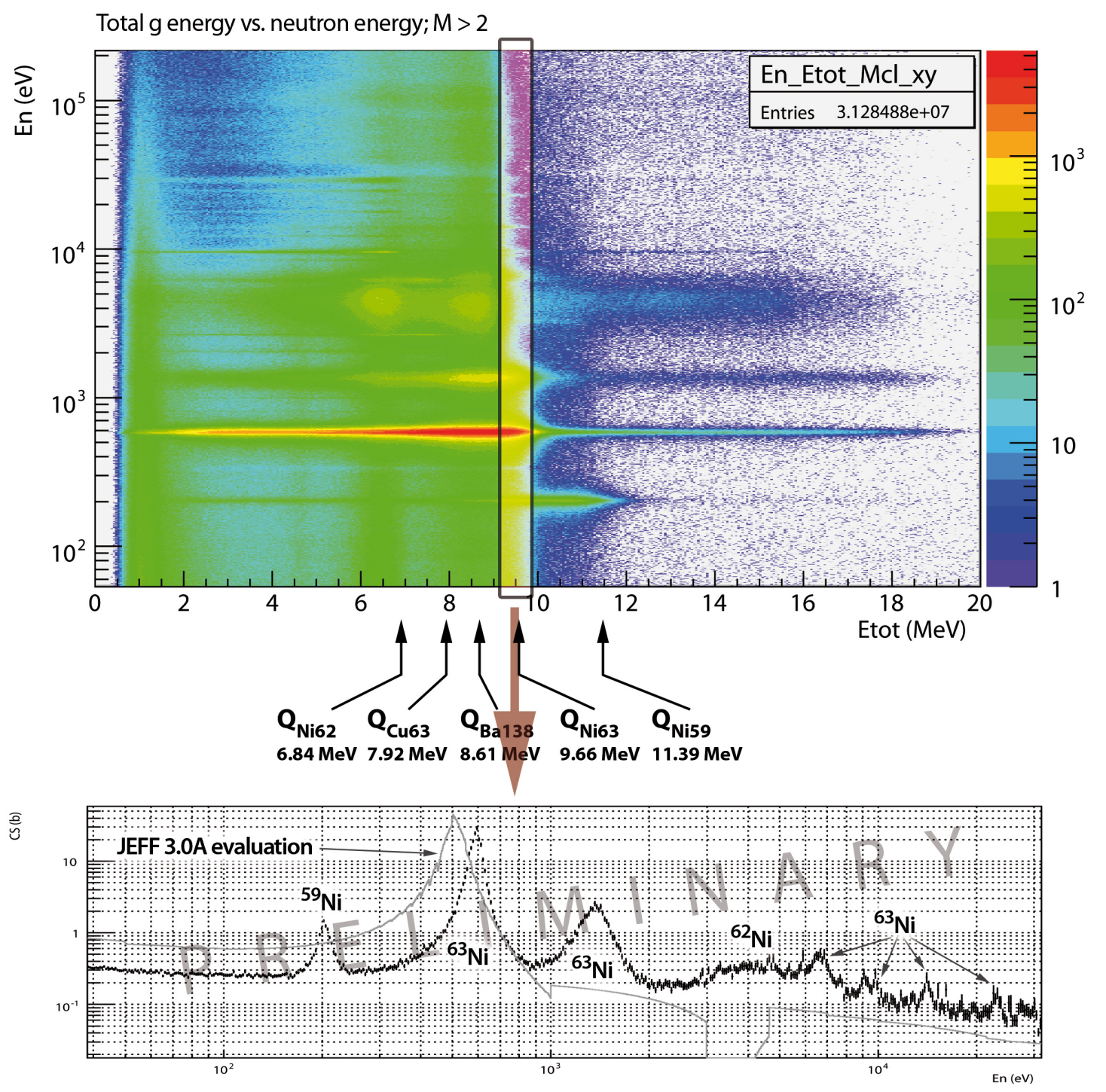

Figure 3: Top: 2D plot of $E_{t o t}$ vs. neutron energy, with multiplicity greater than 2, showing the used data cut in order to get a clean as possible ${ }^{63} \mathrm{Ni}$ CS. Bottom: Preliminary CS vs. the only available data at JEFF 3.0A.

\section{First results}

The ${ }^{63} \mathrm{Ni}(\mathrm{n}, \gamma)$ reaction has a Q-value of $Q_{63} \mathrm{Ni}=9.658 \mathrm{MeV}$. Background from other species in the sample can also be identified via Q-value using the total gamma energy information. Most of the observed background is the result of the main material of the sample, ${ }^{62} \mathrm{Ni}$, and the result of the ${ }^{59} \mathrm{Ni}$ and ${ }^{63} \mathrm{Cu}$ contaminations. Neutron captures on ${ }^{62} \mathrm{Ni}$ and ${ }^{63} \mathrm{Cu}$ have lower Q-values than on ${ }^{63} \mathrm{Ni}\left(Q_{62} \mathrm{Ni}=6.838 \mathrm{MeV}\right.$ and $Q_{63} \mathrm{Cu}=7.916 \mathrm{MeV}$ respectively $)$ and can be discriminated by a cut on the total gamma energy. ${ }^{59} \mathrm{Ni}(\mathrm{n}, \gamma)$, unfortunatly, has Q-value of $Q_{{ }^{99} \mathrm{Ni}}=11.388 \mathrm{MeV}$. But 


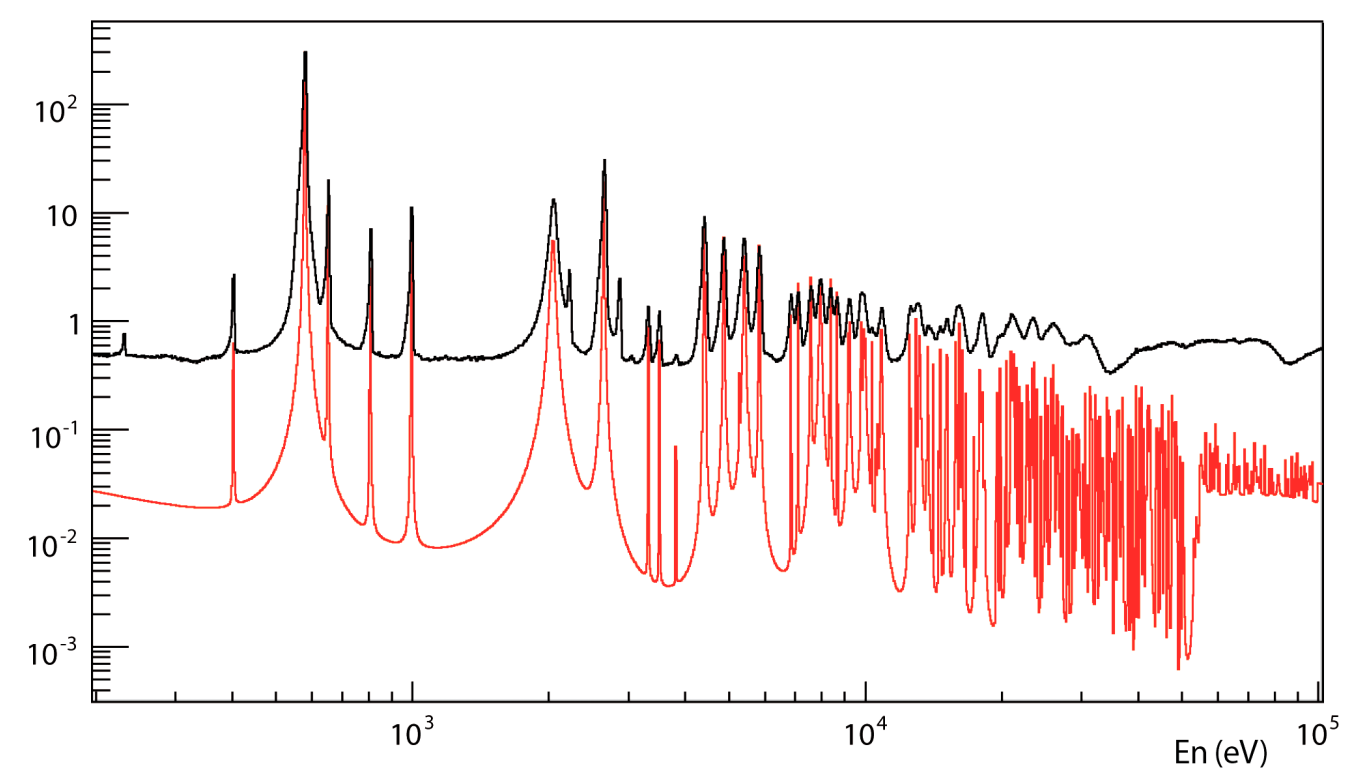

Figure 4: A preliminary, not normalized plot of the ${ }^{63} \mathrm{Cu}$ measurement (black) and JEFF 3.0A data for comparison (red) [8].

the data show, that there is only a significant contribution in the area of the strongest resonance at about $200 \mathrm{eV}$.

Additionally, neutron scattering on ${ }^{62} \mathrm{Ni}$ produces a significant background, which appears at the Q-value of ${ }^{137} \mathrm{Ba}(\mathrm{n}, \gamma)$ at $8.612 \mathrm{MeV}$ due to capture events in the crystals. This background can also be discriminated via $E_{t o t}$ cut. Figure 3 top shows a 2D plot with a rough sketch of the cut. The bottom part shows the current state of the analysis with a preliminary differential cross section.

One major challenge of this analysis is the determination of the efficiency for the used data cut. First, the DICEBOX code was used to create artificial gamma cascades [6]. Second, in order to calculate the fraction of the used data, GEANT3 simulations of the complete detector geometry and with the created cascades were performed [7]. The preliminary result is an efficiency of $6.53 \%$ for the used energy window of 9.2 to $9.7 \mathrm{MeV}$ and multiplicities from 3 to 10 .

Two strong and several smaller peaks could be identified as resonances from ${ }^{63} \mathrm{Ni}(\mathrm{n}, \gamma)$. They are marked in figure 3 bottom.

\section{Further analysis}

The analysis of the ${ }^{63} \mathrm{Ni}$ experiment will be finished soon. Simulation studies with GEANT3, concerning the efficiency of the used data cuts are in the final stages. Additionally the CS will also be determined relative to the Gold standard to verify the results. Follow-up measurements from February 2012 with a $99 \%{ }^{63} \mathrm{Cu}$ sample will provide additional CS data for the $\mathrm{Ni}-\mathrm{Cu}-\mathrm{Zn}$ region. A preliminary plot of the data is shown in figure 4. 


\section{References}

[1] A. M. Alpizar-Vicente, T. A. Bredeweg, E.-I. Esch, U. Greife, R. C. Haight, R. Hatarik, J. M. O’Donnell, R. Reifarth, R. S. Rundberg, J. L. Ullmann, D. J. Vieira, J. M. Wouters. Neutron capture cross section of Ni62 at s-process energies. 77(1) 015806.

[2] I. Dillmann, M. Heil, F. Käppeler, R. Plag, T. Rauscher, F.-K. Thielemann. KADoNiS- The Karlsruhe Astrophysical Database of Nucleosynthesis in Stars. In A. Woehr, A. Aprahamian, eds., Capture Gamma-Ray Spectroscopy and Related Topics, vol. 819 of American Institute of Physics Conference Series (2006), 123-127.

[3] F. Käppeler, R. Gallino, S. Bisterzo, W. Aoki. The s process: Nuclear physics, stellar models, and observations. Reviews of Modern Physics 83 (2011) 157.

[4] M. Pignatari, R. Gallino, M. Heil, M. Wiescher, F. Käppeler, F. Herwig, S. Bisterzo. The Weak s-Process in Massive Stars and its Dependence on the Neutron Capture Cross Sections. Ap. J. 710 (2010) 1557.

[5] R. Reifarth, T. A. Bredeweg, A. Alpizar-Vicente, J. C. Browne, E.-I. Esch, U. Greife, R. C. Haight, R. Hatarik, A. Kronenberg, J. M. O’Donnell, R. S. Rundberg, J. L. Ullmann, D. J. Vieira, J. B. Wilhelmy, J. M. Wouters. Background identification and suppression for the measurement of $(n, \gamma)$ reactions with the DANCE array at LANSCE. Nucl. Instr. Meth. A 531 (2004) 528.

[6] F. Becvár, M. Krticka. Simulations of Gamma Cascades and Modelling Atomic Collision Chains. Journal of Research 105 (2000) 113.

[7] J. Apostolakis. CERN program library long writeup, W5013. Tech. rep., CERN, GEANT library (1993). Http://wwwinfo.cern.ch/asd/geant/.

[8] NEA/EFF, working, group. The JEFF-3.0 Nuclear Data Library. Tech. rep., JEFF Report 19, OECD Nuclear Energy Agency, www.nea.fr/html/dbdata/nds_jefreports/jefreport-19/jefreport-19.pdf (2005). 